\title{
Plantas, polinizadores e algumas articulações da biologia da polinização com a teoria ecológica
}

Plants, pollinators and some contributions from pollination biology to the ecological theory

\author{
Gudryan J. Barônio ${ }^{1,4}$, Artur A. Maciel ${ }^{1}$, Arthur C. Oliveira ${ }^{1}$, Renan O.A.C Kobal ${ }^{1}$, Desirée A.L. Meireles ${ }^{2}$, \\ Vinícius L.G. Brito ${ }^{2} \&$ André R. Rech ${ }^{3}$
}

\begin{abstract}
Resumo
A consolidação em uma área do conhecimento acontece principalmente quando as informações acerca de um determinado fato ou fenômeno são sistematizadas na forma de uma teoria explicativa, capaz de gerar novas hipóteses testáveis. Na biologia da polinização, o teste de diversas hipóteses ecológicas permitiu ampliar o entendimento sobre os processos que originam, mantêm, alteram ou ainda excluem as interações entre plantas e visitantes florais, gerando os padrões observados na natureza. Visando sintetizar esse panorama teórico e oferecer condições para que novas questões relacionadas ao funcionamento de interações de polinização sejam geradas, compilamos aqui um conjunto de 25 hipóteses, ideias e teorias ecológicas que fornecem aporte conceitual para a área. Essas ideias estão relacionadas a aspectos reprodutivos, morfológicos, cognitivos, macroecológicos e de coexistência, de acordo com a especialização das interações entre as plantas e seus polinizadores. Ao apresentarmos essas ideias principais, esperamos promover a utilização de uma abordagem teórico-conceitual explícita no planejamento e desenvolvimento de estudos em biologia da polinização. Concluímos com a expectativa de que essa contribuição direcione os estudos em biologia da polinização no Brasil e contribua para o avanço e internacionalização das pesquisas desenvolvidas no país.

Palavras-chave: Fundamentação teórica, interação planta-polinizador, sucesso reprodutivo, ecologia cognitiva.
\end{abstract}

\begin{abstract}
A particular research area can be significantly improved when information is systematized in explanatory theories able to generate testable hypotheses. In pollination biology, such systematization contributed to several ecological theories and expanded the understanding of the patterns and processes that originated, maintain, or exclude ecological interactions. Here, we compiled a set of 25 ecological ideas that have already received conceptual contribution from pollination biology. These ideas are related to reproduction, morphology, cognition, macroecology and species coexistence. We hope to promote the use of an explicit conceptual and theoretical approach when planning and developing studies in this research field by presenting these main ideas. We conclude that this contribution will boost studies in pollination biology in Brazil and contribute to the advancement and internationalization of such research developed in the country.
\end{abstract}

Key words: Theorical grounds, plant-pollinator interaction, reproductive success, cognitive ecology.

\section{Introdução}

Desde os estudos clássicos de Konrad Sprengel, Charles Darwin, Alfred Wallace e Gregor Mendel, que se dedicaram à busca de respostas para amplas questões ecológicas e evolutivas, os trabalhos envolvendo biologia da polinização fizeram contribuições para a teoria ecológica, especialmente à ecologia funcional (Rech \& Westerkamp 2014). Plantas e polinizadores apresentam diferentes graus de

\footnotetext{
${ }^{1}$ Universidade Federal de Uberlândia, Programa de Pós-graduação em Ecologia e Conservação de Recursos Naturais, Inst. Biologia, Av. Pará 1720, 38405-320, Uberlândia, MG, Brasil.

${ }^{2}$ Universidade Federal de Uberlândia, Inst. Biologia, Av. Pará 1720, 38405-320, Uberlândia, MG, Brasil.

${ }^{3}$ Universidade Federal dos Vales do Jequitinhonha e Mucuri (UFVJM), Faculdade Interdisciplinar de Humanidades, Curso de Licenciatura em Educação do Campo, Campus JK, Rod. MGT 367, Km 583, 5000, Alto da Jacuba, 39100-000, Diamantina, MG, Brasil.

${ }^{4}$ Autor para correspondência: gudryan@gmail.com
} 
interdependência que variam no tempo e no espaço (Blüthgen \& Klein 2011; Vázquez et al. 2012). Essa interdependência, que varia desde interações extremamente especializadas até relações muito generalistas, potencializou a utilização desse tipo de mutualismo como modelo para o estudo de muitas questões ecológicas e evolutivas (Rech \& Brito 2012; Waser \& Ollerton 2006).

Considerando os diferentes aspectos da interação planta-polinizador, Mitchell et al. (2009a) apontaram duas abordagens históricas: (1) estudos focados na observação dos mecanismos florais relacionados à reprodução das plantas e à história natural das relações planta-polinizador e; (2) processos ecológicos e evolutivos subjacentes à polinização. Estas abordagens contribuíram aditivamente e continuam a fomentar o desenvolvimento da biologia da polinização e das teorias ecológicas. Assim, fica clara a união intrínseca de estudos de ecologia e evolução dos sistemas de polinização com a ampliação do entendimento de padrões e processos que originaram, mantêm, alteram, ou ainda excluem mutualismos em seu contexto ambiental (Mitchell et al. 2009a; Biesmeijer et al. 2011).

Conhecer hipóteses ecológicas já testadas no âmbito da biologia da polinização contribui muito para que novas questões relevantes nesse campo de pesquisa sejam formuladas. É sob essa perspectiva que apresentamos aqui uma síntese das principais contribuições da biologia da polinização à teoria ecológica. Compilamos 25 ideias, hipóteses e teorias e com isso esperamos estimular maior articulação entre os conhecimentos da biologia da polinização e a teoria ecológica de maneira ampla, explícita e precisa. Embora alguns dos tópicos abordados ao longo do texto inevitavelmente se adequam a mais de um desses temas, para fins de sistematização as ideias foram agrupadas de acordo com os respectivos temas: I- especialização, II- reprodução, III- morfologia, IV- coexistência, Vmacroecologia e VI- cognição. Baseamos a escolha dos tópicos de acordo com o tema mais abrangente dentro da ecologia da polinização.

\section{I - Especialização}

As angiospermas constituem um dos maiores grupos de seres vivos e apresentam um imenso potencial interativo (Thompson 1994; Waser \& Ollerton 2006). No caso das interações entre as plantas e polinizadores, acreditava-se na prevalência de interações especializadas, ou seja, interações envolvendo poucos parceiros (Waser et al. 2011, para definições dos conceitos de especialização e generalização (veja Ollerton et al. 2007). Nesse sentido, a especialização das interações entre plantas e polinizadores foi defendida por muitos como uma chave central no sucesso da radiação adaptativa das angiospermas (Stebbins 1970; Crepet 1983). Embora existam divergências quanto à universalidade da teoria de síndromes de polinização (Rech et al. 2014), o avanço do debate e as evidências acumuladas permitem hoje uma clareza maior acerca da natureza difusa e contínua do caráter especialização-generalização nas interações de polinização (Fenster et al. 2004; Waser \& Ollerton 2006). Apresentamos abaixo as principais ideias que contribuíram para este entendimento.

\section{Coevolução}

Originalmente designado por Mode (1958) a partir de sistemas parasita-hospedeiros, a teoria da coevolução foi difundida por Ehrlich \& Raven (1964) baseados em um modelo de evolução química entre plantas e insetos. Estes autores notaram que os compostos secundários produzidos por uma planta determinam o uso desta planta como alimento por borboletas. Dessa forma, a diversidade de plantas e dos compostos secundários produzidos por elas contribuem para a diversificação de borboletas e vice-versa. Num sentido mais amplo, coevolução seria então uma mudança evolutiva recíproca dos atributos (ou conjunto de genes) entre duas espécies interagentes, e esse seria o principal processo responsável pela radiação adaptativa dos interagentes.

Do ponto de vista ecológico, todo indivíduo é apto a causar alterações no meio em que vive e interagir com outros indivíduos intra e interespecíficos, mesmo que com diferentes intensidades (Alcock 2009; Ricklefs 2010). Assim, embora muitas vezes sejam utilizados exemplos que citem exclusivamente uma via de mão dupla, a coevolução fatalmente não afeta apenas dois grupos de interagentes, mas sim todo o sistema envolvido (Thompson 1994). Nesse sentido, algumas interações entre plantas e polinizadores possivelmente coevoluiram paralelamente à história evolutiva dos herbívoros, um importante grupo que gera pressões seletivas às plantas (Sletvold \& Grindeland 2008; Barônio 2012, mas veja Campbell 2015). A interação planta-herbívoro também é apontada como grande mecanismo de diversificação das angiospermas (Leimu et al. 2012) e afeta diretamente as interações planta- 
polinizador, que exibe reflexos coevolutivos na atratividade dos polinizadores e nos sistemas reprodutivos (Para detalhes veja: Johnson et al. 2015).

$\mathrm{Na}$ literatura da área encontra-se inclusive a defesa de que a seleção das plantas pelos polinizadores é tão variável, pouco intensa e com tantas flutuações no tempo e espaço que isoladamente não direcionaria a diversificação das angiospermas (Thompson 1994; Crepet \& Nicklas 2009). Entretanto, a coevolução entre planta e polinizador foi apontada como uma das principais causas da rápida especiação das angiospermas (Johnson 1998; Lunau 2004). A teoria coevolutiva dos mosaicos geográficos prevê a plasticidade de interações e organismos interagentes de acordo com a variação temporal e geográfica (Thompson 1994). Essa característica estabelecida pela teoria infere que pode haver interação de diferentes estádios adaptativos (Thompson 1994), assim essa continuidade não levaria necessariamente à especialização. De acordo com essa perspectiva mutualística, os processos coevolutivos que resultassem em especialização promoveriam em alguns casos mecanismos de isolamento reprodutivo (Bhattacharyay \& Drossel 2005). Assim, baseada em mutualismos, a percepção das relações coevolutivas entre plantas e polinizadores tem sido questionada por evidências recentes de que os genes que promovem o sucesso reprodutivo das plantas parecem, em geral, ter um efeito negativo sobre os polinizadores e vice-versa. De acordo com essa ideia, apesar da manutenção das parcerias mutualísticas através da reciprocidade de interesses, relações mutualísticas se desenvolvem por meio de processos antagonistas (Chamberlain \& Rudgers 2012; Santamaría \& Rodríguez-Girónes 2015).

\section{"Beco sem Saída evolutivo"}

Sob a ótica da especialização, determinados atributos podem levar organismos a melhorar seu desempenho a curto prazo. Porém, a longo prazo essa especialização entre espécies interagentes pode levar ao estreitamento de nicho, tornando as espécies mais vulneráveis às mudanças no ambiente, levando a um "ponto final" irreversível no processo evolutivo e uma consequente extinção (Kassen 2002; Agnarsson et al. 2006; Kelley \& Farrell 1988; Tripp \& Manos 2008), a isso denomina-se "beco sem saída evolutivo". Dessa forma, as linhagens que evoluíram em associação exclusiva com outros organismos, ou ainda com um número reduzido de interações em relação a outras linhagens, teriam maiores probabilidade de extinção. A hipótese evolutiva de beco sem saída iniciou-se com Futuyma \& Moreno (1988) sugerindo que a frequência de interações entre generalistas que originam interações especialistas durante o tempo evolutivo deve ser maior que a rota inversa.

A evolução da autopolinização a partir de sistemas de polinização cruzada foi bastante discutida como um possível beco sem saída da evolução. As características relacionadas à autopolinização aparentemente representam uma solução a curto prazo que favorece a colonização de uma nova área sem polinizadores ou a perda dos serviços de polinização, mas que a longo prazo poderia levar à extinção (Takebayashi \& Morrell 2001, mas veja: Igic \& Busch 2013). Esse exemplo foi parcialmente descartado após a descoberta de que a maioria dos taxa apomíticos são facultativos e que mesmo sendo raras, algumas populações apresentam aumento na diversidade genética através de reprodução sexuada (Bashaw et al. 1970, mas veja também: Dall'agnol \& Schifino-Wittmann 2005; van Dijk \& Vijverberg 2005). Entretanto, a partir da afirmação de que a autopolinização é irreversível e que as linhagens apomíticas têm aumento nas taxas de extinção (Barrett 2013), a discussão tem sido retomada com intenção de entender o que pode ser considerado um beco sem saída na evolução dos sistemas reprodutivos de acordo com o tipo de especialização, seja ela com presença de interações (polinização cruzada) ou não (apomixia entre outras) (Tripp \& Manos 2008; Wright et al. 2013).

\section{Competição}

Apesar da possível vulnerabilidade da especialização, algumas interações de polinização permanecem extremamente especializadas ao longo do tempo (Rech \& Brito 2012). Certamente existem várias vantagens na especialização, entre elas o aumento na eficiência da polinização e no fluxo gênico intraespecífico (Johnson \& Steiner 2000). Para que essas vantagens sejam efetivas, historicamente se assumiu um cenário de constante competição por polinizadores, que podem ser limitados. Nesse sentido, as plantas seriam sempre polinizadas aquém da possibilidade (veja MoodyWeis \& Heywood, 2001), hipótese que ficou conhecida como hipótese do polinizador limitante. A floração assincrônica entre espécies de plantas polinizadas pelo mesmo grupo de polinizadores 
e a partição de polinizadores entre espécies de plantas sincrônicas estão entre os exemplos de padrões que sugeririam mecanismos competitivos no âmbito da biologia da polinização (Mitchell et al. 2009b). Além da competição por polinizadores, a competição entre polinizadores também gera pressões sobre as interações. Por exemplo, Balfour et al. (2015) realizaram experimentos de exclusão de abelhas de grande e médio porte que competem no ambiente. Neste estudo observaram que as abelhas de grande porte limitavam e interferiam na atividade das abelhas menores, além de elevar significativamente a disponibilidade de recurso floral na condição de baixa competição. Dessa forma foi possível concluir que a competição por exploração é uma forte influência na formação e manutenção da comunidade estudada (Balfour et al. 2015).

\section{Síndromes de polinização}

Considerando que polinizadores podem ser agentes seletivos, e que grupos de polinizadores apresentam preferências similares, surgiu a hipótese de síndromes de polinização (Faegri \& Pijl 1979). De acordo com essa hipótese, grupos de plantas que compartilham os mesmos grupos de polinizadores tem um conjunto de características florais convergentes, permitindo a previsão dos polinizadores a partir destas. Essa previsibilidade seria dada de acordo com o conjunto de necessidades da planta em relação aos visitantes e das preferências do visitante em relação aos atributos florais (veja Schiestl \& Johnson 2013). Caso esse cenário fosse constante, seria possível deduzir o grupo de polinizadores baseando-se nas características florais. Existem estudos que corroboram essa expectativa da hipótese de síndromes de polinização (Pauwn 2006; Armbruster et al. 2011). Por outro lado, uma abordagem quantitativa, desenhada para avaliar a congruência de flores acopladas às síndromes de polinização, verificou que dificilmente o fenótipo floral se encaixa perfeitamente no conjunto de atributos específicos esperados para um grupo de polinizadores (Ollerton et al. 2009). Assim, considerando que essas características florais são diretamente associadas de modo a qualificar um visitante como polinizador e que o processo de coevolução dessas interações é contínuo e variável, é aceitável que interações com acoplamentos perfeitos sejam raros. No entanto, mesmo considerando o grupo de polinizadores morfologicamente mais próximo das características florais, ainda assim, a previsibilidade do conceito de síndromes parece ficar abaixo de 50\% (Ollerton et al. 2009). Além disso, os estudos com redes de interação também têm demonstrado uma ampla prevalência, pelo menos do ponto de vista taxonômico, de interações generalistas na natureza. Isto vai de encontro ao que seria predito caso as síndromes fossem claramente demarcáveis e a irradiação adaptativa a partir do processo de coevolução fosse o único motriz da diversificação das angiospermas (Freitas et al. 2014). Entretanto, é importante ressaltar alguns pontos em que há possibilidade de explicação dessa baixa previsibilidade das síndromes. Embora cada síndrome possua características próprias e compartilhem em alguns casos os mesmos caracteres, a importância deles pode ter diferentes estímulos nos respectivos polinizadores. Além do mais, essa coevolução entre plantas e polinizadores acontece em um contexto espaço-tempo no qual ocorrem outras interações, por exemplo entre as plantas e herbívoros, que pode também direcionar as espécies a se ajustarem de forma equiparada entre os diferentes interagentes (Junker et al. 2007; Junker \& Blüthgen 2010).

\section{II - Coexistência}

A coexistência de espécies é definida pela sua sobreposição espaço-temporal. No entanto, o que define essa capacidade de sobreposição espaçotemporal são as características de cada comunidade interagindo com o ambiente (Giacomini 2007). A interação de algumas espécies com o ambiente pode torná-lo mais ou menos estável para a colonização de outras espécies (Connell \& Slatyer 1977; Padilla \& Pugnaire 2006). Apresentaremos aqui algumas noções ecológicas acerca da coexistência de espécies já testadas com plantas e polinizadores.

\section{Distúrbio intermediário}

Para explicar diferenças na diversidade de espécies entre locais distintos, Connell (1978) propôs a hipótese do distúrbio intermediário, argumentando que um nível moderado de perturbação criaria as máximas condições de partição dos recursos e portanto, as condições para mais espécies coexistirem (Tilman 1982; Amarasekare 2003). No caso da polinização, Cartar (2005) sugere que distúrbios mais intensos aumentam a riqueza de plantas e visitantes, o que poderia favorecer serviços de polinização. Ele comparou, em ambiente boreal, locais com diferentes porcentagens de floresta: nenhuma 
árvore remanescente $(0 \%)$, poucas árvores remanescentes (10-20\%), quantidade moderada de árvores remanescentes (50-75\%) e, como controle, usou outra área onde nenhuma árvore foi removida. Nesse experimento, a remoção intermediária das árvores (50-75\%) apresentou a maior abundância de abelhas, de plantas visitadas por abelhas, flores e maior diversidade de aparatos florais. Entretanto, a credibilidade desta hipótese tem sido bastante questionada e uma revisão envolvendo mais de 100 trabalhos com distúrbios intermediários mostrou que são raras as sobreposições entre o máximo da diversidade das espécies e o nível intermediário da perturbação (Mackey \& Currie 2001).

\section{Facilitação por efeito de sobreposição ou "efeito magnético"}

Esta ideia baseia-se no princípio de que conjuntos mais diversos de flores podem criar condições nas quais o serviço de polinização seja otimizado, ao invés de promoverem a competição entre as espécies. Sendo assim, a sincronia de floração entre espécies poderia facilitar a atração de polinizadores ao mesmo tempo em que evitaria a predação floral intensiva de uma espécie em particular (Harder \& Johnson 2005; Mickeliunas et al. 2006). Com um experimento de translocação de plantas de Raphanus raphanistrum L., (Brassicaceae) para áreas com floração ativa de outras três, duas e uma espécie nativa, e para uma área exclusiva, Ghazoul (2006) observou que a planta apresenta menor número de polinizadores e de sementes por frutos quando isolada do que nas condições de co-ocorrência. Além disso, a riqueza de visitantes florais foi maior quando as quatro espécies co-ocorriam, o que reafirma o efeito facilitador da coexistência neste caso.

\section{Teoria Neutra}

Essa ideia defende que os padrões de riqueza e abundância de espécies poderiam ser explicados majoritariamente por processos neutros, e os processos ecológicos, como as interações, seriam menos relevantes na montagem de comunidades (Hubbell 2001). Recentemente tal conceito foi abordado nos estudos das interações entre plantas e polinizadores, e há um foco em identificar o conjunto de regras responsáveis pela estrutura e topologia de redes de interações (Diamond 1975; Freitas et al. 2014). Vázquez (2005) utilizou um modelo nulo simples para comparar 17 redes mutualísticas (12 interações de polinização e cinco de planta-frugívoro). O intuito foi verificar se a topologia dessas redes mutualísticas poderia ser interpretada como determinada a partir de "combinações de espécies proibidas" ou seria similar a uma estrutura gerada por interações aleatórias como proposto pela teoria neutra. As redes simuladas a partir dos fundamentos da teoria neutra revelaram uma topologia muito próxima ao observado na natureza. Assim, considerando a premissa da teoria da neutralidade de que todas as espécies são ecologicamente equivalentes e interagem através de um mecanismo de loteria (Hubbell 2006), Vázquez (2005) concluiu que o modelo neutro fornece uma interpretação alternativa dos padrões observados nas redes de interações ecológicas. Essa combinação aleatória das interações pode ser o melhor modo para explicar a topologia de algumas redes mutualísticas observadas, principalmente nas regiões temperadas. Por outro lado, estudos feitos na região neotropical, têm demonstrado que as interações proibidas como aquelas entre plantas e polinizadores que não ocorrem no mesmo tempo e/ou espaço pode determinar a estrutura das redes de modo não neutro (Vizentin-Bugoni et al. 2014; Maruyama et al. 2014).

\section{Espaço livre de inimigo}

Um espaço livre de inimigos é definido como o espaço (e.g., localidade geográfica ou micro-habitat) onde ocorre redução populacional, extinção ou inacessibilidade de inimigos naturais ou competidores de uma determinada espécie (Jeffries \& Lawton 1984). Estudando as vespas do figo, Al-Beidh et al. (2012) trazem um bom exemplo da teoria de espaço livre de inimigos aplicada no contexto de biologia da polinização. Nesse estudo, eles identificaram três camadas de flores com diferentes níveis de parasitismo dentro dos sicônios. Nas duas camadas mais externas as vespas polinizadoras competem por frutos em desenvolvimento com vespas não polinizadoras e parasitoides que acessam o interior do fruto através da parede sem precisar adentrá-lo. Nesse sistema, a diferenciação de nicho espacial de espécies competidoras devido às diferenças na acessibilidade permite a coexistência de polinizadores e não polinizadores. A camada mais interna, à qual as vespas não polinizadoras e parasitoides tem pouco acesso, é muito mais intensamente ocupada pelas vespas polinizadoras que se desenvolvem então em um espaço livre de inimigos (Al-Beidh et al. 2012). 


\section{Coexistência mediada por predador}

Em locais onde não existe pressão por predação, um forte competidor pode excluir os demais. No entanto, a existência de predadores pode criar condições que viabilizem a coexistência de competidores (Kullberg \& Ekman 2000). Por exemplo, o favorecimento de uma espécie na disputa por recurso alimentar, pode ser alterada quando parasitoides exercem um efeito negativo na habilidade competitiva do hospeiro (Lebrun \& Feener 2002). Gonzálvez et al. (2013) mostraram em um estudo com interação de três níveis tróficos que a presença de um predador beneficia o nível trófico mais basal. Nesse estudo, Melastoma malabathricum L. (Melastomataceae) é polinizada por abelhas e produz substâncias que atraem formigas tecelãs (Oecophylla smaragdina Fabricius 1775). Dentre os polinizadores, dois gêneros (Xylocopa - grandes e eficientes e Nomia - pequenas e ineficientes) representam 99\% de visitas. Nesse sistema, a formiga $O$. smaragdina é o predador e constrói ninhos e forrageia nessas plantas. Com experimentos de exclusão os autores constataram que a presença da formiga aumenta o sucesso reprodutivo de M. malabathricum, pois as abelhas menores e menos eficientes evitam seletivamente plantas com formigas enquanto as abelhas maiores e mais eficientes são indiferentes a esta condição (Gonzálvez et al. 2013). Este estudo mostra claramente que a interação formigaplanta concilia a utilização dos recursos de $M$. malabathricum por duas espécies de abelha.

\section{Similaridade limitante e deslocamento de caráter}

Assumindo a premissa de que a similaridade de caracteres entre as espécies possa causar competição no uso comum de recursos entre as espécies, MacArthur \& Levins (1967) formularam a hipótese da similaridade limitante. Nesse contexto, Maruyama et al. (2014) observaram em plantas polinizadas por beija-flores que as interações planta-polinizadores se organizam em módulos associados com a especialização morfológica e a ocupação de hábitat. Dessa forma, ocorrem coexistências mesmo havendo uma similaridade morfológica relacionada ao compartilhamento de recursos pelos diferentes módulos associados na rede de interações. $\mathrm{O}$ deslocamento de caráter é uma variação na morfologia floral de espécies induzida pela simpatria, que permite a coexistência mantendo ou até mesmo aumentando a aptidão (Miyake \& Inoue
2003; Pfennig \& Pfennig 2010). O próprio Darwin (1859) deixou claro que a divergência de caracteres era importante para a origem e coexistência de novas espécies, uma vez que a competição poderia causar tal divergência e, uma vez acontecendo entre populações, essas alterações morfológicas poderiam causar especiação (Pfennig \& Pfennig 2010; Benadi et al. 2013). Em um estudo com espécies polinizadas por morcegos, Muchhala \& Potts (2007) concluíram que a quantidade de pólen coespecífico depositado pelos morcegos era diretamente proporcional às diferenças no comprimento da língua dos animais. Esse exemplo mostra que a competição interespecífica pela transferência de pólen pode ser reduzida quando há diferença nas características florais, as quais podem ser induzidas via deslocamento de caracteres (Muchhala \& Potts 2007). Dessa forma, embora existam exemplos de espécies que coexistem utilizando recursos similares, em condições em que há limitação de recursos, as espécies precisariam diferir suficientemente para que isso causasse uma alteração no nicho que permitisse a utilização concomitante do ambiente (Abrams 1983). Embora essas duas hipóteses estejam, não apenas, relacionadas com características morfológicas das espécies, o entendimento de como ocorre a similaridade limitante e deslocamento de caráter também pode explicar a coexistência das espécies interagentes. Assim, essas duas hipóteses também podem ser relacionadas às hipóteses coexistenciais apresentadas acima.

\section{III - Reprodução}

O funcionamento dos mecanismos reprodutivos de plantas despertou inúmeras questões científicas ao longo da história e, como em várias outras áreas, Darwin foi um dos pioneiros na formulação destas questões (Richards 1986). Seus trabalhos iniciais foram essenciais para o entendimento sobre auto-incompatibilidade, depressão endogâmica, heteromorfia e outras características reprodutivas em plantas (Darwin 1876, 1877). Apresentamos aqui, entre estas questões, algumas relacionadas com a segurança reprodutiva, geitonogamia, competição masculina e a escolha feminina.

\section{Segurança reprodutiva e Efeito Allee}

A ideia de segurança reprodutiva debate as condições nas quais mecanismos que garantam a reprodução de plantas, como a autopolinização e a apomixia ofereceriam vantagens adaptativas em 
determinados ambientes. Dada a independência de polinizadores, Baker (1955) propôs que uma planta hermafrodita teria maior probabilidade de se estabelecer após um evento de dispersão a longas distâncias conquanto tivesse reprodução uniparental (veja mais detalhes em Pannell et al. 2015). Esta expectativa, conhecida como "Lei de Baker" foi expandida para maior ocorrência de autogamia em plantas pioneiras (Pannell \& Barrett 1998). Assim, tanto a autogamia quanto a apomixia podem ser entendidas como estratégias atuantes em pequenas populações naturais (Cheptou 2004) e na manutenção de espécies com baixas densidades atráves de mecanismos de autopolinização (Morgan et al. 2005), que atenuariam o Efeito Allee. Esse efeito pode ser definido como uma correlação positiva entre o valor adaptativo individual e o número ou densidade de coespecíficos (Stephens 1999). Também é sugerida uma diferenciação entre o efeito Allee em componentes isolados do valor adaptativo e o efeito Allee demográfico, que se manifesta no nível total do valor adaptativo, considerando todos os seus componentes (Stephens 1999). Dessa forma, o efeito deve ser visto como o saldo líquido dos efeitos positivos e negativos da densidade no valor adaptativo total. Um teste do efeito Allee relacionado com a biologia da polinização de plantas foi feito com a planta invasora, Lilium formosanum, que se reproduz tanto pela polinização por mariposas quanto por autogamia (Rodger et al. 2013). Através de emasculação e suplementação de pólen, os autores demonstraram que a importância da autogamia aumenta com a diminuição do tamanho populacional e aumento do isolamento. Assim, verificaram que a autogamia compensa o déficit dos serviços de polinização e também a limitação de pólen dirigido pela força do Efeito Allee.

\section{Amplitude da distribuição de espécies influenciada pelo sistema reprodutivo}

A abundância de indivíduos de uma espécie é variável, geralmente sendo maior nas populações centrais da área de ocorrência do que nas populações periféricas (Hegenveld \& Haeck 1982). Isso porque na direção do centro para a periferia da distribuição ocorreria uma diminuição progressiva dos parâmetros de nicho adequados para a espécie (Brown 1984). Porém, gradientes descontínuos mais complexos podem produzir padrões de distribuição e abundância de forma mais abrupta do que o esperado (Lawton 1993). Por ser sensível ao ambiente, a biomassa dos indivíduos em populações periféricas tende a ser menor e, com isso, o número de flores por indivíduos também cai (Weiner et al. 2009). Assim, os recursos apresentados por estas plantas tornam-se menos atraentes para polinizadores, que por essa razão, podem se tornar menos constantes em suas rotas de forrageio. Isso aumentaria a deposição inadequada ou a limitação de pólen causando diminuição da reprodução em populações marginais já que os níveis de polinização cruzada cairiam (Goulson \& Wright 1998; Aguilar et al. 2006).

A redução das oportunidades de cruzamento com outros indivíduos em populações periféricas favorece a seleção de alelos que aumentam a autogamia, causando variações na morfologia floral, desenvolvimento e fisiologia que promovem segurança reprodutiva (Fausto et al. 2001; Elle \& Carney 2003). Essa hipótese é sustentada por muitos estudos que relacionam a taxa de autogamia em populações que ocorrem em áreas periféricas às outras populações predominantemente alogâmicas (Baker 1955; Levin 2012).

\section{Geitonogamia e dilema da planta}

O "dilema da planta" foi proposto a partir de um conflito evolutivo em que o aumento no número de flores aumentaria a atratividade para os polinizadores, mas ao mesmo tempo aumentaria também o número de visitas em flores de uma mesma planta durante a mesma rota de forrageio do polinizador. Devido a esse aumento na autopolinização, esse conflito proporciona maior geitonogamia, que é a fração de autofertilização entre flores de uma mesma planta (Hessing 1988; Klinkhamer \& de Jong 1993), em especial em plantas onde a reprodução vegetativa (clonal) é constante e elevada (Barrett 2015; Vallejo-Marín et al. 2010).

Por outro lado, os custos associados à geitonogamia podem ser baixos, já que um polinizador é fisicamente capaz de visitar apenas uma quantidade limitada de flores em cada forrageio, o que permitiria o depósito de grãos de pólen exógenos nos estigmas do indívíduo em questão devido ao comportamento do polinizador (Klinkhamer et al. 1994). Além disso, abelhas geralmente apresentam certa constância floral, ou seja, visitam flores de uma mesma espécie em diferentes indivíduos a cada evento de forrageio (Chittka et al. 1999). Ainda, as consequências da geitonogamia diferem entre plantas auto-compatíveis e autoincompatíveis. No primeiro caso, geitonogamia conduz a altas 
taxas de autofertilização, mas nas plantas que são autoincompatíveis, ela apenas reduz o sucesso reprodutivo pela deposição imprópria de pólen nos estigmas e desperdício de grãos de pólen (de Jong et al. 1993; Klinkhamer \& de Jong 1993).

\section{Seleção sexual (competição masculina e escolhas femininas)}

A seleção sexual pode operar intrassexualmente, através de competição, ou interssexualmente, por escolha de parceiros. Ambos os mecanismos ocorrem em plantas e daí derivam a hipótese da competição masculina e a hipótese da escolha feminina (Stephenson 1983). Além disso, deve-se também levar em consideração as respostas femininas a essas estratégias competitivas masculinas, o que pode influenciar a paternidade através de mecanismos que permitam uma otimização no número e qualidade da prole (Birkhead \& Pizzari 2002). A competição masculina no contexto das plantas ocorre entre grãos de pólen de diferentes indivíduos ou grãos de pólen de um mesmo macho no nível da flor, mesmo que exista uma limitação de pólen no nível de planta (Parker 1970). Apesar disso, Cocucci et al. (2014) realizaram um estudo que demonstrou o primeiro exemplo de competição pré-polinização direta entre polinárias de Asclepias L., evidenciando que devido aos apêndices pontiagudos, as polinárias interagem entre elas ao longo da suceção de polinárias aderidas ao corpo do polinizador.

A hipótese de escolha feminina considera que selecionar os grãos de pólen traz menos custos às fêmeas do que o posterior aborto de zigotos ou embriões. Além disso, a escolha feminina também pode evitar autofertilizações ou ainda fertilizações por indivíduos geneticamente próximos. Mesmo não existindo o controle direto sobre a identidade dos seus parceiros, características que aumentam a visita de polinizadores podem aumentar o número de grãos de pólen advindos de diferentes indivíduos (Willson \& Burley 1983). Esses podem ser selecionados por sistemas de autoincompatibilidade, uma característica que ocorre em aproximadamente $60 \%$ das espécies de plantas (Hiscock \& Kues 1999). É interessante ressaltar que esses mecanismos de seleção de machos e fêmeas podem levar a um conflito sexual através da divergência entre traços ótimos para as funções masculinas e femininas (Bedhomme et al. 2009; Parker 1979; Chapman et al. 2003; Schärer et al. 2014), o que é esperado, pois na maior parte das angiospermas o hermafroditismo é comum. Revisões mostram que a seleção sexual, associada com a desigualdade consistente no sucesso reprodutivo, ocorre principalmente durante as fases pós-polinização (Marshall \& Folsom 1991; Snow \& Lewis 1993). No entanto, a seleção também pode ocorrer antes da polinização, através da otimização da atração ou da maior adesão de grãos de pólen ao corpo do polinizador. Evolutivamente, observa-se um acoplamento entre planta e o agente polinizador que permite otimizar as funções florais masculinas e femininas (Delph \& Ashman 2006). A distinção entre competição masculina e escolha feminina é dificultada pelo pequeno limiar de reconhecimento nas interações pós-polinização onde, de forma geral, as influências entre as estruturas reprodutivas masculinas e femininas ocorrem de forma simultânea (Marshall \& Folsom 1991; Stanton 1994).

\section{IV - Morfologia floral}

A flor é a interface de comunicação entre plantas e polinizadores (Harder \& Barrett 2006; Brito et al. 2014). Nessa interação, a morfologia da flor influencia e é influenciada pelos visitantes florais (e.g., Jones \& Reithel 2001; Moré et al. 2012). Embora os sistemas de polinização sejam bastante diversos, algumas adaptações se tornaram tão eficientes que são mantidas em diferentes espécies por convergência evolutiva (Herrera 1996). Nesta seção serão apresentadas algumas idéias que contextualizam a ecologia da polinização às características morfológicas dos organismos interagentes.

\section{Hipótese de Berg}

A estabilidade evolutiva dos caracteres florais foi explorada inicialmente por Berg (1960), ao propor que estes teriam maior integração entre si do que os caracteres vegetativos e que isso seria mais evidente em plantas com polinização especializada (Ordano et al. 2008). De acordo com a hipótese, dentro de sistemas especializados, dada necessidade de manutenção do fino ajuste entre as morfologias das flores e de seus polinizadores, a variação na morfologia dos caracteres florais tornaria-se desacoplada da variação na morfologia de caracteres vegetativos (Armbruster et al. 1999, 2009, 2011). Análises comparando variação das morfologias floral e vegetativa corroboraram a hipótese de Berg (Conner \& Sterling 1996; Pélabon et al. 2011; Lázaro \& Totland 2014). Para testar a hipótese, Conner \& Sterling (1996) selecionaram 
cinco espécies com morfologia floral semelhante e com possibilidade de medir os mesmos caracteres e encontraram uma maior correlação dentro dos grupos de caracteres (florais e vegetativos). Em um estudo com Dalechampia scandens, (Euphorbiaceae), submetida a diferentes condições de disponibilidade de nutrientes e irradiação, observou-se que a variação fenotípica nas brácteas atrativas associadas à inflorescência era dissociada dos padrões de variação nos caracteres vegetativos e associada às características reprodutivas. Esses resultados condizem com a hipótese de Berg, uma vez que essas brácteas possuem funções indiretas que intermediam a sinalização aos polinizadores e também protegem as flores (Hansen et al. 2007; Pélabon et al. 2011).

\section{Dilema do pólen}

Ocorre em função da dualidade reprodutivoalimentar na pressão seletiva exercida sobre os grãos de pólen (Harder \& Thomson 1989; Lunau et al. 2014). Dentre as estruturas diretamente ligadas à polinização, as anteras são alvo de grande pressão seletiva dos polinizadores, uma vez que o pólen é o recurso que obrigatoriamente deve entrar em contato com o corpo do visitante para que possa haver polinização (Thorp 2000; Schiestl \& Johnson 2013). Porém, o pólen também é ativamente coletado como alimento por alguns polinizadores, como as abelhas e algumas borboletas (Westerkamp 1996; Thorp 2000). Assim, em plantas polinizadas por insetos coletores de pólen, estratégias que envolvessem defesas contra esse tipo de coleta tenderiam a ser evolutivamente favorecidas, no sentido de minimizar o consumo alimentar em detrimento do aumento da função reprodutiva (Thorp 2000; Santamaría e Rodriguez-Girones 2015).

Recentemente, Lunau et al. (2014) demonstraram que alguns dos grãos de pólen de Alcea rosea possuem pequenos espinhos que dificultam o acúmulo do pólen nas corbículas das abelhas. As flores desta espécie também ofereçam néctar, o qual mantem a atratividade mesmo quando a coleta de pólen pode não ser mais vantajosa. Essa proteção mecânica contra a coleta ativa dos grãos de pólen, no entanto, não impede a fixação dos grãos no corpo do visitante garantindo o transporte para a polinização (Lunau et al. 2014).

\section{Divisão de trabalho}

No mesmo contexto, dado que o pólen é utilizado como alimento pelos polinizadores (especialmente abelhas), uma forma de atraí-los sem comprometer todos os grãos de pólen é a compartimentalização deste em diferentes partes florais. Considera-se aqui a heteranteria, na qual ocorre uma diferenciação funcional nas anteras, geralmente pelo tamanho, cor, posicionamento, direcionamento do poro e tipo de pólen produzido (Vallejo-Marín et al. 2009). Assim, após uma sugestão feita pelos irmãos Fritz e Hermann Müller de que haveria uma divisão de trabalho entre essas anteras com morfologias diferentes, Darwin (1862) cunhou o termo "divisão de trabalho". Essa hipótese prediz que anteras menores teriam função de oferecer o recurso aos polinizadores, enquanto as anteras maiores teriam função reprodutiva (Luo et al. 2008; Lunau et al. 2014). Essa diferença em tamanho e posicionamento permite que haja anteras preferencialmente manipuladas pelos polinizadores e outras que, embora menos manipuladas, exportam maior quantidade de pólen para polinização (Vallejo-Marín et al. 2009).

\section{V - Macroecologia}

As hipóteses desenvolvidas na macroecologia, bem como as relacionadas à ecologia de comunidades, também oferecem um arcabouço teórico para o desenvolvimento de estudos em biologia da polinização. Nesta seção, apresentamos questões macroecológicas cujas hipóteses foram também testadas no âmbito da biologia da polinização.

\section{Estabilização da composição e do funcionamento de ecossistemas via incremento em biodiversidade}

De acordo com essa ideia, a estabilização da composição e funcionamento de ecossistemas por incremento de biodiversidade poderia se dar por duas razões principais: (1) pela maior expressão do papel funcional via efeito aditivo ou facilitador das espécies agregadas ou (2) pela maior robustez de grupos funcionais mais ricos em espécies (Lawton \& Brown 1993; Naeem \& Li 1997; Loreau et al. 2001). No âmbito da biologia da polinização, o papel estabilizador da biodiversidade foi testado utilizando-se uma série temporal de meio século de interações entre macieiras e seus polinizadores. No trabalho foi utilizado um cenário de mudanças climáticas e previu-se que populações de macieiras com maior diversidade de polinizadores permaneceriam mais ajustadas fenologicamente ao seu grupo de polinizadores e com a função de polinização melhor garantida ao longo do tempo (Bartomeus 
et al. 2013). Como na região temperada, onde foi realizado o estudo, as abelhas apresentam complementaridade fenológica no tempo de emergência, os autores previram que a maior riqueza de abelhas aumentaria o tempo em que alguma delas estaria disponível para polinizar, diminuindo o risco de flores de macieira com fenologia deslocada por aquecimento global não serem polinizadas. Os autores encontraram uma mudança fenológica grande no período de floração das macieiras ao longo do tempo de estudo, sendo que, conforme esperado, essa mudança foi melhor tamponada nos locais de maior diversidade de abelhas (Bartomeus et al. 2013).

\section{Gradiente latitudinal de interações}

Esta ideia prevê que dada maior estabilidade e menor sazonalidade da região tropical, existiria um gradiente positivo no nível de especialização das interações ecológicas em direção ao equador (Schemske et al. 2009). No entanto, a sustentação para essa hipótese ainda é bastante controversa, em grande parte pelo imenso vazio de informações sobre interações ecológicas no nível de comunidade que paira sobre a região tropical do planeta (Ollerton \& Cranmer 2002; Schemske et al. 2009; Ollerton et al. 2011). Apresentaremos aqui, para o campo da biologia da polinização, uma evidência de que a especialização nas interações planta-polinizador seja: (1) inversamente correlacionada, (2) diretamente correlacionada e (3) não correlacionada com a latitude.

O primeiro trabalho configura um exemplo de que as interações planta-polinizador nos trópicos seriam menos especializadas. $\mathrm{O}$ estudo foi realizado por Schleuning et al. (2012) a partir da análise de redes de interação. Os autores utilizaram um índice que mediria a especialização em redes interativas (representação matemática das interações ecológicas no nível de comunidade) e concluíram que a especialização seria provavelmente uma resposta a menor diversidade de plantas do que uma compartimentalização de nichos (Schleuning et al. 2012). A tendência oposta foi ilustrada, entre outros estudos com polinização, no trabalho também com redes interativas de Dalsgaard et al. (2011) utilizando beija-flores e flores no Neotrópico. Estes autores observaram que o gradiente latitudinal explicava em torno de $20 \%$ da variação no nível de especialização das redes, evidenciando que as interações tropicais foram mais especializadas. O terceiro exemplo, na verdade confunde-se com a hipótese nula dos exemplos anteriores, qual seja, de que não há um gradiente. Estudando interações entre flores e polinizadores, embora Ollerton \& Cranmer (2002) tenham encontrado inicialmente uma relação negativa entre latitude e especialização, quando os autores controlaram a análise pelo esforço de coleta o padrão simplesmente desapareceu. Essa ideia tem apoio também no fato de que os nichos das espécies tropicais, comparativamente às temperadas, em geral também não parecem ser mais estreitos (Vázquez \& Stevens 2004).

\section{Legado histórico}

Essa hipótese prevê que as variações no clima e na paisagem ocorridas no passado de uma localidade influenciam as características e os processos contemporâneos das espécies ou suas interações. Embora aparentemente óbvia, essa hipótese carece de mecanismos para ser testada propriamente, e muitas vezes sua utilização foi considerada como a invocação do "fantasma da evolução passada" (Linares 1997). Com o aperfeiçoamento das ferramentas de biologia molecular, climatologia e da modelagem matemática, tornou-se possível obter mais informações sobre o passado das linhagens biológicas e sobre os possíveis cenários nos quais elas evoluíram. Modelagens climáticas permitem simulações de como seriam e como variaram ao longo do tempo alguns descritores do clima como, por exemplo, temperatura e precipitação (Sandel et al. 2011). Com base nesse tipo de dados, a dinâmica climática do passado já foi relacionada com padrões de distribuição e diversidade de espécies (Svenning \& Skov 2007; Sandel et al. 2011), demografia e estrutura genética de populações (Cabanne et al. 2007), e no âmbito da biologia da polinização com a estrutura de redes de interações complexas entre plantas e polinizadores (Dalsgaard et al. 2011, 2013). No estudo de Dalsgaard et al. (2013), a modelagem do clima pretérito contribuiu significativamente para explicar os padrões de modularidade e aninhamento em redes de interação entre plantas e polinizadores. Os autores encontraram uma relação negativa entre modularidade e estabilidade climática do Pleistoceno, além de uma relação positiva entre o mesmo preditor e o aninhamento, ou seja, áreas mais instáveis ao longo do Quaternário contribuíram para redes mutualísticas contemporâneas menos modulares e mais aninhadas (Dalsgaard et al. 2013). 


\section{VI - Cogniç̧ão}

Além das funções reprodutivas, a flor possui função de atrair os visitantes, especialmente os polinizadores, através de sinais específicos como cores, odores, formatos e simetria (Lehrer et al. 1995). Dessa forma, a incorporação de tais atributos cognitivos nas teorias ecológicas tem sido uma via importante para a compreensão dos processos envolvidos na interação plantapolinizador (Brito et al. 2014). Aqui apresentamos um compilado das ideias relacionadas à interface das interações planta-polinizador a partir dos aspectos cognitivos dos visitantes florais, principalmente àquelas relacionados à cor. Porém, ressaltamos que essas ideias também se aplicam a outros atributos, como odor, forma e simetria.

\section{Exclusão sensorial}

Cada grupo de visitante floral tem sua própria forma de percepção do ambiente devido às diferenças em seus aparatos cognitivos (Brito et al. 2014). A hipótese de exclusão sensorial, ou hipótese de canal privado, prevê que os sinais florais que potencializam a interação planta-polinizador são evidentes, na maioria das vezes, apenas para um grupo restrito de organismos (Raguso 2008a). Assim, os demais grupos de animais que não conseguem perceber esses sinais seriam excluídos cognitivamente das possibilidades de interação com a planta. Chen et al. (2009) ao examinarem o mutualismo obrigatório entre Ficus semicordata Miq. e vespas do figo observaram que um composto incomum produzido pela planta é o responsável pela atração específica do polinizador. Em uma análise utilizando uma planta com variação intraespecífica na coloração das flores, Bergamo et al. (2015) demonstrou que flores brancas era visitadas por abelhas e beija-flores enquanto flores rosas, por configurarem um canal privado de comunicação com beija-flores (abelhas seriam menos visualmente estimuladas por esta cor), estariam excluindo sensorialmente as abelhas.

\section{Mimetismo batesiano}

Aplicado aos estudos com polinização, o mimetismo batesiano ocorreria quando flores que não oferecem recursos, ou recursos pouco compensadores, se beneficiam por emitir sinais semelhantes aos sinais emitidos por flores gratificantes, de forma que os agentes biológicos não conseguissem diferenciá-los (Anderson et al. 2005; Newman et al. 2012). O mimetismo batesiano tem sido o fenômeno mais evocado para explicar as interações em Orchidaceae, o táxon com maior número de espécies que não oferecem recursos aos polinizadores (Jersáková et al. 2006). Parece haver relações diretas entre o êxito reprodutivo das orquídeas e a distância com as espécies gratificantes que emitem sinais semelhantes (Johnson et al. 2003; Peter \& Johnson 2008). Dentre os sinais imitados pelas orquídeas, os principais são odores, cores e formas, sendo a cor aparentemente o fator mais importante (Jersáková et al. 2012). Uma determinada espécie de polinizador pode, inclusive, ter indivíduos com preferências de cor diferentes em função das suas experiências com as cores das flores gratificantes ou não na comunidade local onde vive (Newman et al. 2012).

\section{Mimetismo de pólen}

Essa hipótese prevê que existam estruturas ou partes florais com características similares ao pólen ou anteras e que isso aumentaria a atratividade floral. Em geral, manchas na cor amarela UV-absorvente nas flores funcionam na atração de abelhas polinizadoras, enquanto o pólen de fato estaria escondido. Dessa forma, a similaridade ocorre entre a cor do pólen e/ ou antera e a cor de uma pequena área central da flor (Heuschen et al. 2005). O pólen é um recurso muito procurado pelas abelhas fêmeas por servir como fonte proteica para manutenção de seus ninhos (Proctor et al. 1996). No caso do mimetismo de pólen, as plantas mímicas imitam os sinais de um modelo geral (a cor dos grãos de pólen) e não os sinais de uma determinada espécie, e essa semelhança pode aumentar ou substituir os sinais visuais associados ao próprio pólen, e assim, potencializar maiores chances de visita (Lunau 2000). Heuschen et al. (2005) mostraram que, no sistema de visão em cores de Bombus terrestris, flores e inflorescências com simetria radial e flores zigomórficas possuem a cor do centro menos diversificada do que a cor da periferia. Além disso, a cor do centro nessas situações também foi muito semelhante à cor de pólen. Nesse mesmo trabalho, abelhas inexperientes mostraram uma preferência por flores com duas cores em detrimento de flores com apenas uma cor, sendo aquelas com cor do centro semelhante à cor do pólen mais visitadas do que aquelas com outras cores no centro. 


\section{Exploração de preferências inatas}

Ao se tratar de flores que enganam seus polinizadores, o mimetismo não é o único mecanismo que pode explicar esse tipo de interação. $\mathrm{O}$ sistema sensorial dos polinizadores possuem propriedades inatas que podem afetar a percepção e a preferência por estímulos (Schaefer \& Ruxton 2009; Lunau et al. 2011). Desta forma, as plantas podem explorar o sistema sensorial dos polinizadores ao emitir um sinal inatamente preferido por ele e esse mecanismo pode ser muito mais comum que o mimetismo. Assim, orquídeas polinizadas por engano sexual podem explorar os insetos não só pela semelhança com um sinal específico de outra espécie mas também pela exploração do sistema sensorial (Raguso 2008b). De fato, em algumas orquídeas que não oferecem nenhum tipo recurso podem ocorrer visitas sem a presença de um modelo específico (Jersáková et al. 2006). Outro exemplo de exploração de preferências inatas involve a interação entre flores do gênero Aristolochia e moscas. Tradicionalmente essas flores tem sido encaixadas dentro da síndrome de sapromiiofila ou micromiiofilia mediada pelo odor floral (Faegri $\&$ van der Pijl 1979). Porém, um estudo mostrou que a espécie Aristolochia rotunda explora as preferências inatas de moscas ao produzir odores semelhantes à hemolinfa de hemípteros da ordem Miridae da qual geralmente essas moscas se alimentam (Oelschlägel et al. 2015).

\section{Gradiente unidirecional de saturação}

A hipótese do gradiente unidirecional de saturação, no contexto da ecologia da polinização, prevê que as flores apresentariam um gradiente de saturação que direcionaria o polinizador para o recurso, geralmente da borda para o centro das flores. Lunau (1990) realizou uma série de experimentos com cenários artificiais utilizando $B$. terrestris recém emergidas, a fim de verificar quais as preferências inatas de cores dessas abelhas. Esse pesquisador registrou que a pureza espectral da cor da corola é um fator crucial na atração à distância para as flores. Em experimentos semelhantes Lunau et al. (1996) mostraram que a diferença de cor entre a corola e o centro da flor estão correlacionadas positivamente com a frequência de visitas, ou seja, quanto maior a distância entre essas cores, mais visitas. Quando se considerou apenas as visitas de abelhas que contactaram as antenas nas flores artificiais houve uma grande influência do sentido em que apontava a diferença de cor. Assim, a saturação foi o atributo mais importante e as flores com a cor do centro mais saturada foram as mais visitadas, indicando uma preferência inata a este padrão de cor floral (Lunau et al. 1996).

\section{Forrageio ótimo}

Essa ideia iniciou com o trabalho de MacArthur \& Pianka (1966) tratando da economia energética e o forrageamento no ambiente e inserindo alguns fatores que determinariam o comportamento de forrageio em animais. Polinizadores estão entre os animais mais estudados com relação a essa teoria, pela facilidade em se calcular a quantidade de calorias do néctar (Fonseca et al. 2015) e seguir seu comportamento de vôo (Pyke 1978). De acordo com a hipótese de forrageio ótimo, o tamanho do território de forrageio de um polinizador territorialista deveria maximizar seu ganho energético, ou seja, render o máximo de néctar coletado com o mínimo de perda energética com o seu deslocamento ou a defesa desse território (McDade \& Weeks 2004). Entre os exemplos de evidências produzidas no contexto da biologia da polinização em relação à hipótese do forrageio ótimo, sabe-se que abelhas partindo de flores onde encontraram néctar tendem a visitar flores próximas a esta com mais frequência do que quando encontravam uma flor sem néctar (Waddington \& Holden 1979). Pyke (1978) demonstrou que a decisão de beija-flores em deixar uma inflorescência é função do número de flores já visitadas, do número total de flores e do volume de néctar obtido na última visita. No caso das abelhas, experimentos mostram que a constância na oferta do recurso é um elemento de decisão importante, havendo preferência por flores com recursos menos variáveis mesmo quando esses não são os mais energéticos (Real 1981). Com um experimento natural, Gonzálvez et al. (2012), demonstraram que abelhas do gênero Nomia tendem a evitar plantas com a presença de formigas predadoras, enquanto abelhas maiores do gênero Xylocopa não suscetíveis a predação por formigas, modificavam seu comportamento de coleta, passando a visitar preferencialmente as plantas com formigas. Considerados os controles necessários para o experimento, os autores concluíram em favor da hipótese de forrageio ótimo como causa para o comportamento das abelhas, o que por consequência tem efeitos dramáticos sobre o padrão de cruzamentos realizado por cada indivíduo da planta em questão (Gonzálvez et al. 2012). 


\section{Considerações Finais}

Com base no panorama aqui apresentado, a biologia da polinização tem sido um importante patamar para o desenvolvimento de diversas contribuições relacionadas ao escopo da teoria ecológica. É importante conhecer essas teorias para formulação de novas questões que possam levar ao maior avanço do conhecimento. Fica claro também o ganho teórico que se tem ao se articular as ideias específicas em teste com o contexto maior no qual elas se inserem. Com a crescente qualidade das publicações e consequente disputa por espaço em periódicos internacionais, vemos uma necessidade dessa articulação para a internacionalização dos trabalhos científicos nacionais, bem como para o fortalecimento dos veículos de publicação brasileiros. Esses objetivos serão alcançados somente com um aumento da qualidade das publicações, e expomos aqui algumas das principais ideias, hipóteses e teorias já testadas e relacionadas à biologia da polinização. Baseados apenas em interações planta-polinizador, apresentamos tópicos relacionados à especialização, reprodução, morfologia, coexistência, padrões macroecológicos e aos processos cognitivos dessas interações. Esperamos que as teorias, hipóteses e ideias sintetizadas aqui possam esclarecer, integrar e aprofundar as questões investigadas nos estudos de biologia da polinização.

\section{Agradecimentos}

Os autores encarecidamente agradecem aos amigos Paulo Eugênio Oliveira, Felipe W. Amorim, Pietro K. Maruyama, Carlos E. P. Nunes (Coquinho), Jeferson Vizentin-Bugoni e Pedro J. Bergamo, a ajuda na fase de levantamento de hipóteses relevantes. Agradecemos também aos revisores, as contribuições ao manuscrito durante a fase de revisão, em especial ao Prof. Erich Arnold Fischer. GJB agradece a bolsa de Doutorado CAPES/UFU 2013-2017 e VLGB agradece a bolsa PNPD/PPGBV-UFU. Esse trabalho é resultado de um trabalho desenvolvido na disciplina "Fronteiras em Biologia da Polinização" oferecida pelo Programa de Pós-graduação em Biologia Vegetal pela Universidade Federal de Uberlândia.

\section{Referências}

Abrams, P. 1983. The theory of limiting similarity. Annual Review of Ecology and Systematics 14: 359-376.

Aguilar, R.; Ashworth, L.; Galetto, L. \& Aizen, M.A. 2006. Plant reproductive susceptibility to habitat fragmentation: review and synthesis through a metaanalysis. Ecology Letters 9: 968-980.

Agnarsson, I.; Avile, L.S.; Coddington, J. A. \& Maddison, W. 2006. Sociality in Theridiid spiders: repeated origins of an evolutionary dead end. Evolution 60: 2342-2351.

Al-Beidh, S.; Dunn, D.W.; Power, S.A. \& Cook, J.M. 2012. Parasites and mutualism function: measuring enemy-free space in a fig-pollinator symbiosis. Oikos 121: 1833-1839.

Alcock, J. 2009. Animal Behaviour. Sinauer Associates. Arizona. 546p.

Amarasekare, P. 2003. Competitive coexistence in spatially structured environments: a synthesis. Ecology Letters 6: 1109-1122.

Anderson, B.C.; Johnson, S.D. \& Carbutt, C. 2005. Exploitation of a specialized mutualism by a deceptive orchid. American Journal of Botany 92: 1342-1349.

Armbruster, W.S.; Hansen, T.F.; Pélabon, C.; PérezBarrales, R. \& Maad, J. 2009. The adaptive accuracy of flowers: measurement and microevolutionary patterns. Annals of Botany 103: 1529-1545.

Armbruster, W.S.; Gong, Y-B. \& Huang, S-Q. 2011. Are Pollination "Syndromes" Predictive? Asian Dalechampia Fit Neotropical Models. The American Naturalist 178: 135-143.

Armbruster, W.S.; Stilio, V.S. Di; Tuxill, J.D.; Flores, T.C. \& Velásquez Runk, J.L. 1999. Covariance and decoupling of floral and vegetative traits in nine neotropical plants: a re-evaluation of Berg's correlation-pleiades concept. American Journal of Botany 86: 39-55.

Baker, HG. 1955. Self-compatibility and establishment after 'long distance' dispersal. Evolution 9: 347-348.

Balfour, N.J.; Gandy, S. \& Ratnieks, F.L.W. 2015. Exploitative competition alters bee foraging and flower choice. Behavioral Ecology and Sociobiology 69: 1731-1738. DOI: 10.1007/s00265-015-1985-y.

Barônio, G.J. 2012. Leaf hairiness reduces herbivory of young and mature leaves of Qualea multiflora Mart. in Brazilian Savanna. Neotropical Biology and Conservation 7: 122-128.

Barrett, S.C.H. 2013. The evolution of plant reproductive systems: how often are transitions irreversible? Proceedings of the Royal Society B: Biological Sciences 280: 20130913. DOI:10.1098/ rspb.2013.0913

Barrett, S.C.H. 2015. Influences of clonality on plant sexual reproduction. Proceedings of the National Academy of Sciences 112: 8859-8866.

Bartomeus, I.; Park, M.G.; Gibbs, J.; Danforth, B.N.; Lakso, A.N. \& Winfree, R. 2013. Biodiversity ensures plant-pollinator phenological synchrony against climate change. Ecology Letters 16: 1331-1338.

Bashaw, E.C.; Hovin, A. \& Holt, E.C. 1970. Apomixis, its evolutionary significance and utilization in plant 
breeding. In: International Grassland Congress, 11, 1970. Proceedings of the XI International Grassland Congress held at Surfers Paradise, Queensland. Pp. 245-248.

Bedhomme, S.; Bernasconi, G.; Koene, J.M.; Lankinen, A.; Arathi, H.S.; Michiels, N.K. \& Anthes, N. 2009. How does breeding system variation modulate sexual antagonism? Biology Letters 5: 717-720.

Benadi, G.; Blüthgen, N.; Hovestadt, T. \& Poethke, H.J. 2013. When can plant-pollinator interactions promote plant diversity? The American naturalist 182: 131-46.

Benitez-Vieyra, S.; Moré, M. \& Amorim, F. 2014. Seleção fenotípica mediada por polinizadores. In: Rech, A.R.; Agostini, K.; Oliveira, P.E.A.M. \& Machado, I.C. Biologia da Polinização. Editora Projeto Cultural, Rio de Janeiro. 524p.

Bergamo, P.J.; Rech, A.R.; Brito, V.L.G. \& Sazima, M. In press. Flower colour and visitation rates of Costus arabicus support the "bee avoidance" hypothesis for red-reflecting hummingbirdpollinated flowers. Functional Ecology. DOI: 10.1111/1365-2435.12537

Berg, R.L. 1960. The ecological significance of correlation pleiades. Evolution 14: 171-180.

Bessa-Gomes, C.; Legendre, S. \& Clobert, J. 2004. Allee effects, mating systems and the extinction risk in populations with two sexes. Ecology Letters 7: 802-812.

Bhattacharyay, A. \& Drossel, B. 2005. Modeling coevolution and sympatric speciation of flowers and pollinators. Physica A: Statistical Mechanics and its Applications 345: 159-172.

Biesmeijer, J.C.; Sorensen, P.B. \& Carvalheiro, L.G. 2011. How pollination ecology research can help answer important questions. Journal of Pollination Ecology 4: 68-73.

Birkhead, T.R. \& Pizzari, T. 2002. Postcopulatory sexual selection. Nature Reviews Genetics 3: 262-273.

Blüthgen, N. \& Klein, A.M.M. 2011. Functional complementarity and specialisation: the role of biodiversity in plant-pollinator interactions. Basic and Applied Ecology 12: 282-291.

Brito, V.L.G.; Telles, F. \& Lunau, K. 2014. Ecologia cognitiva da polinização. In: Rech, A.R.; Agostini, K.; Oliveira, P.E.A.M. \& Machado, I.C. Biologia da Polinização. Editora Projeto Cultural, Rio de Janeiro. 524p.

Brown, J.H. 1984. On the relationship between abundance and distribution of species. American Naturalist 124: 255-279.

Cabanne, G.S.; Santos, F.R. \& Miyaki, C.Y. 2007. Phylogeography of Xiphorhynchus fuscus (Passeriformes, Dendrocolaptidae): vicariance and recent demographic expansion in southern Atlantic forest. Biological Journal of the Linnean Society 91: 73-84.
Campbell, S.A. 2015. Ecological mechanisms for the coevolution of mating systems and defence. New Phytologist 205: 1047-1053.

Cartar, R.V. 2005. Short-term effects of experimental boreal forest logging disturbance on bumble bees, bumble \& bee-pollinated flowers and the bee-flower match. Biodiversity \& Conservation 14: 1895-1907.

Chamberlain, S.A. \& Rudgers, J.A. 2012. How do plants balance multiple mutualists? Correlations among traits for attracting protective bodyguards and pollinators in cotton (Gossypium). Evolutionary Ecology 26: 65-77.

Chapman, T.; Arnqvist, G.; Bangham, J. \& Rowe, L. 2003. Sexual conflict. Trends in Ecology \& Evolution 18: 41-47.

Chen, C.; Song, Q.; Proffit, M.; Bessière, J.M.; Li, Z. \& Hossaert-McKey, M. 2009. Private channel: a single unusual compound assures specific pollinator attraction in Ficus semicordata. Functional Ecology 23: 941-950.

Cheptou, P.O. 2004. Allee effect and self-fertilization in hermaphrodites: reproductive assurance in demographically stable populations. Evolution 58: 2613-2621.

Chittka, L.; Thomson, J.D. \& Waser, N.M. 1999. Flower constancy, insect psychology, and plant evolution. Naturwissenschaften 86: 361-377.

Cocucci, A.A.; Marino, S.; Baranzelli, M.; Wiemer, A.P. \& Sérsic, A. 2014. The buck in the milkweed: evidence of male-male interference among pollinaria on pollinators. New Phytologist 203: 280-286.

Coley, P.D. \& Kursar, T.A. 2014. On tropical forests and their pests. Science 343: 35-36.

Connell, J.H. \& Slatyer, R.O. 1977. Mechanisms of succession in natural communities and their role in community stability and organization. American Naturalist 111: 1119-1144.

Connell, J. H. 1978. Diversity in tropical rain forests and coral reefs. Science 199: 1302-1310.

Conner, J.K. \& Sterling, A. 1996. Selection for independence of floral and vegetative traits: evidence from correlation patterns in five species. Canadian Journal of Botany 74: 642-644.

Crepet, W.L. 1983. The role of pollination in the evolution of the angiosperms. In: Real, L. (ed.). Pollination biology. Academic Press, Orlando. Pp. 29-50.

Crepet, W.L. \& Niklas, K.J. 2009. Darwin's second "abominable mystery": why are there so many angiosperm species? American Journal of Botany: Darwin Bicentenial 96: 366-381.

Dall'agnol, M. \& Schifino-Wittmann, M.T. 2005. Apomixia, genética e melhoramento de plantas. Revista Brasileira de Agrociências 11: 127-133.

Dalsgaard, B.; Magård, E.; Fjeldså, J.; Martín-González, A.M.; Rahbek, C.; Olesen, J.; Ollerton, J.; Alarcón, R.; Araujo, A.C.; Cotton, P.A.; Lara, C.; Machado, 
C.G.; Sazima, I.; Sazima, M.; Timmermann, A.; Watts, S.; Sandel, B.S.; Sutherland, W.J. \& Svenning, J.C. 2011. Specialization in planthummingbird networks is associated with species richness, contemporary precipitation and quaternary climate-change velocity. PLoS One 6: e25891.

Dalsgaard, B.; Trøjelsgaard, K.; González, A.M.M.; Nogués-Bravo, D.; Ollerton, J.; Petanidou, T.; Sandel, B.; Schleuning, M.; Wang, Z.; Rahbek, C.; Sutherland, W.J.; Svenning, J.C. \& Olesen, J.M. 2013. Historical climate-change influences modularity and nestedness of pollination networks. Ecography 36: 1331-1340.

Darwin, C.R. 1859. On the origin of species by means of natural selection, or the preservation of favoured races in the struggle for life. John Murray, London. 502p.

Darwin, C.R. 1862. On the various contrivances by which British and foreign orchids are fertilised by insects. John Murray, London. 366p.

Darwin, C.R. 1877. The different forms of flowers on plants of the same species. John Murray, London. 352p.

De Jong, T.J.; Waser, N.M. \& Klinkhamer, P.G.L. 1993. Geitonogamy: the neglected side of selfing. Trends in Ecology and Evolution 8: 321-325.

Delph, L.F. \& Ashman, T.L. 2006. Trait selection in flowering plants: how does sexual selection contribute? Integrative and Comparative Biology 46: 465-472.

Diamond, J.M. 1975. Assembly of species communities. In: Diamond, J.M. \& Cody, M. (eds.). Ecology and evolution of communities Harvard University Press. Harvard. Pp. 342-444.

Ehrlich, P.R. \& Raven, P.H. 1964. Butterflies and plants: a study in coevolution. Evolution 18: 586-608.

Elle, E. \& Carney, R. 2003. Reproductive assurance varies with flower size in Collinsia parviflora (Scrophulariaceae). American Journal of Botany 90: 888-896.

Faegri, K. \& van der Pijl, L. 1979. The principles of pollination ecology. Pergamon Press, Oxford. 242p.

Fausto, J.A.; Eckhart, V.M. \& Geber, M.A. 2001. Reproductive assurance and the evolutionary ecology of self-pollination in Clarkia xantiana (Onagraceae). American Journal of Botany 88: 1794-1800.

Fenster, C.B.; Armbruster, W.S; Wilson, P.; Thomson, J.D. \& Dudash, M.R. 2004. Pollination syndromes and floral specialization. Annual Review of Ecology, Evolution and Systematics 35: 375-403.

Fonseca, L.C.N.; Vizentin-Bugoni J.; Rech A.R. \& Alves, M.A.S. 2015. Plant-hummingbird interactions and temporal nectar availability in arestinga from Brazil. Anais de Acadêmia Brasileira de Ciências 87: 21632175.

Fox, J.W. 2013. The intermediate disturbance hypothesis should be abandoned. Trends in Ecology \& Evolution 28: 86-92.

Freitas, L.; Vizentin-Bugoni, J.; Wolowski, M.; Souza, L.M.T. \& Varassin, I.G. 2014. Interações planta- polinizador e a estruturação das comunidades. In: Rech, A.R.; Agostini, K.; Oliveira, P.E.A.M. \& Machado, I.C. Biologia da Polinização. Editora Projeto Cultural, Rio de Janeiro. 524p.

Futuyma, D.J. \& Moreno, G. 1988. The evolution of ecological specialization. Annual Review of Ecology and Systematics 19: 207-234.

Ghazoul, J. 2006. Floral diversity and the facilitation of pollination. Journal of Ecology 94: 295-304.

Giacomini, H.C. 2007. Os mecanismos de coexistência de espécies como vistos pela teoria ecológica. Oecologia Brasiliensis 11: 521-543.

Gonzálvez, F.G.; Santamaría, L.; Corlett, R.T. \& Rodríguez-Gironés, M.A. 2013. Flowers attract weaver ants that deter less effective pollinators. Journal of Ecology 101: 78-85.

Goulson, D. \& Wright, N.P. 1998. Flower constancy in the hoverflies Episyrphus balteatus (Degeer) and Syrphus ribesii (L.) (Syrphidae). Behavioral Ecology 9: 213-219.

Hansen, T.F.; Pélabon, C. \& Armbruster, W.S. 2007. Comparing variational properties of homologous floral and vegetative characters in Dalechampia scandens: testing the Berg hypothesis. Evolutionary Biology 34: 86-98.

Harder, L.D. \& Barrett, S.C. 2006. Ecology and evolution of flowers. Oxford University Press, New York. 370p.

Harder, L.D. \& Thomson, J.D. 1989. Evolutionary options for maximizing pollen dispersal of animal-pollinated plants. The American Naturalist 133: 323-344.

Harder, L.D. \& Johnson, S.D. 2005. Adaptive plasticity of floral display size in animal-pollinated plants. Proceedings of the Royal Society B: Biological Sciences 272: 2651-2657.

Hengeveld, R. \& Haeck, J. 1982. The distribution of abundance. I. Measurements. Journal of Biogeography 9: 303.

Herrera, C.M. 1996. Floral traits and plant adaptation to insect pollinators: a devil's advocate approach. In: Lloyd, D.G. \& Barrett, S. (eds.). Floral biology: studies on floral evolution in animal-pollinated plants. Chapman and Hall, New York. Pp 65-87.

Hessing, M.B. 1988. Geitonogamous pollination and its consequences in Geranium caespitosum. American Journal of Botany 75: 1324-1333.

Heuschen, B.; Gumbert, A. \& Lunau, K. 2005. A generalised mimicry system involving angiosperm flower colour, pollen and bumblebees' innate colour preferences. Plant Systematics and Evolution 252: 121-137.

Hiscock, S.J. \& Kües, U. 1999. Cellular and molecular mechanisms of sexual incompatibility in plants and fungi. International Review of Cytology 193: 165-295.

Hubbell, S.P. 2001. The unified neutral theory of biodiversity and biogeography. Princeton University Press, Princeton. 392p. 
Hubbell, S.P. 2006. Neutral theory and the evolution of ecological equivalence. Ecology 87: 1387-1398.

Igic, B. \& Busch, J.W. 2013. Is self-fertilization an evolutionary dead end? New Phytologist 198: 386397.

Jeffries, M.J. \& Lawton, J.H. 1984. Enemy free space and the structure of ecological communities. Biological Journal of the Linnean Society 23: 269-286.

Jersáková, J.; Johnson, S.D. \& Kindlmann, P. 2006. Mechanisms and evolution of deceptive pollination in orchids. Biological Reviews 81: 219-235.

Jersákova, J.; Jürgens, A.; Smilauer, P. \& Johnson, S.D. 2012. The evolution of flora mimicry: identifying traits that visually attract pollinators. Functional Ecology 26:1381-1389.

Johnson, S.D.; Linder, H.P. \& Steiner, K.E. 1998. Phylogeny and radiation of pollination systems in Disa (Orchidaceae). American Journal of Botany 85: 402-411

Johnson, S.D. \& Steiner, K.E. 2000. Generalization versus specialization in plant pollination systems. Trends Ecology and Evolution 15: 140-143.

Johnson, S.D.; Peter, C.I.; Nilsson, L.A. \& Ågren, J. 2003. Pollination success in a deceptive orchid is enhanced by co-occurring rewarding magnet plants. Ecology 84: 2919-2927.

Johnson, M.T.J.; Campbell, S.A. \& Barrett, S.C.H. 2015. Evolutionary interactions between plant reproduction and defense against herbivores. Annual Review of Ecology, Evolution, and Systematics 46: 191-213.

Jones, K.N. \& Reithel, J.S. 2001. Pollinator-mediated selection on a flower color polymorphism in experimental populations of Antirrhinum (Scruphulariaceae). American Journal of Botany 88: 447-454.

Junker, R.; Chung, A.Y.C. \& Blüthgen, N. 2007. Interaction between flowers, ants and pollinators: additional evidence for floral repellence against ants. Ecological Research 22: 665-670.

Junker, R. \& Blüthgen, N. 2010. Floral scents repel facultative flower visitors, but attract obligate ones. Annals of Botany. 105: 777-782.

Kassen, R. 2002. The experimental evolution of specialists, generalists, and the maintenance of diversity. Journal of Evolutionary Biology 15: 173-190.

Kelley, S.T. \& Farrell, B.D. 1998. Is specialization a dead end? The phylogeny of host use in Dendroctonus bark beetles (Scolytidae). Evolution 52: 1731-1743.

Klinkhamer, P.G.L. \& de Jong, T.L. 1993. Attractiveness to pollinators: a plant's dilemma. Oikos 66: 180-184.

Klinkhamer, P.G.L.; de Jong, T.L. \& Metz, J.A.J. 1994. Why plants can be too attractive - a discussion of measures to estimate male fitness. Journal of Ecology 82: 191-194.

Kullberg, C. \& Ekman, J. 2000. Does predation maintain tit community diversity? Oikos 89: 41-45.
Lawton, J.H. \& Brown V.K. 1993. Redundancy in ecosystems. In: Schulze, E.D. \& Mooney, H.A. (eds.). Biodiversity and ecosystem function. SpringerVerlag, Berlin. Pp. 255-270.

Lawton, J.H. 1993. Range, population abundance and conservation. Trends in Ecology \& Evolution 8: 409-413.

Lázaro, A. \& Totland, O. 2014. The influence of floral symmetry, dependence on pollinators and pollination generalization on flower size variation. Annals of Botany 114: 157-165.

LeBrun, E.G. \& Feener, D.H. 2002. Linked indirect effects in ant-phorid interactions: impacts on ant assemblage structure. Oecologia 133: 599-607.

Lehrer, M.; Horridge, G.A.; Zhang, S.W. \& Gadagkar, R. 1995. Shape vision in bees: innate preference for flower-like patterns. Philosophical Transaction Royalty Society B 347: 123-137.

Leimu, R.; Muola, A.; Laukkanen, L.; Kalske, A.; Prill, N. \& Mutikainen, P. 2012. Plant-herbivore coevolution in a changing world. Entomologia Experimentalis et Applicata 144: 3-13.

Levin, D.A. 2012. Mating system shifts on the trailing edge. Annals of Botany 109: 613-620.

Linares, M. 1997. The ghost of mimicry past: laboratory reconstitution of an extinct butterfly "race". Heredity 78: 628-635.

Loreau, M.; Naeem, S.; Inchausti, P.; Bengtsson, J.; Grime, J.P. \& Hector, A. 2001. Biodiversity and ecosystem functioning: current knowledge and future challenges. Science 294: 804-808.

Lunau, K. 1990. Colour saturation triggers innate reactions to flower signals: flower dummy experiments with bumblebees. Journal of Comparative Physiology A 166: 827-834.

Lunau, K. 2000. The ecology and evolution of visual pollen signals. Plant Systematics and Evolution 222: 89-111.

Lunau, K. 2004. Adaptive radiation and coevolution pollination biology case studies. Organisms, Diversity \& Evolution 4: 207-224.

Lunau, K.; Papiorek, S.; Eltz, T. \& Sazima, M. 2011. Avoidance of achromatic colours by bees provides a private niche for hummingbirds. Journal of Experimental Biology 214: 1607-1612.

Lunau, K.; Piorek, V.; Krohn, O. \& Pacini, E. 2014. Just spines-mechanical defense of malvaceous pollen against collection by corbiculate bees. Apidologie 46: 144-149.

Lunau, K.; Wacht, S. \& Chittka, L. 1996. Colour choices of naive bumble bees and their implications for colour perception. Journal of Comparative Physiology A 178: 477-489.

Luo, Z.; Zhang, D. \& Renner, S.S. 2008. Why two kinds of stamens in buzz-pollinated flowers? Experimental support for Darwin's division-of-labour hypothesis. Functional Ecology 22: 794-800. 
MacArthur, R. \& Levins, R. 1967. The limiting similarity, convergence, and divergence of coexisting species. The American Naturalist 101: 377-385.

MacArthur, R.H. 1972. Geographical ecology: patterns in the distribution of species. Princeton University Press, Princeton, Princeton. 288p.

MacArthur, R.H. \& Pianka, E.R. 1966. On optimal use of a patchy environment. The American Naturalist 100: 603-609.

Mackey, R.L. \& Currie, D.J. 2001. The diversitydisturbance relationship: is it generally strong and peaked? Ecology 82: 3479-3492.

Marshall, D.L. \& Folsom, M.W. 1991. Mate choice in plants: an anatomical to population perspective. Annual Review of Ecology and Systematics 22: 37-63.

Maruyama, P.K.; Vizentin-Bugoni, J.; Oliveira, G.M.; Oliveira, P.E. \& Dalsgaard, B. 2014. Morphological and spatio-temporal mismatches shape a neotropical savanna plant-hummingbird network. Biotropica 46: 740-747.

McDade, L. \& Weeks, J. 2004. Nectar in hummingbirdpollinated neotropical plants I: patterns of production and variability in 12 species. Biotropica 36: 196-215.

Mickeliunas, L.; Pansarin, E.R. \& Sazima, M. 2006. Biologia floral, melitofilia e influência de besouros Curculionidae no sucesso reprodutivo de Grobyaamherstiae Lindl. (Orchidaceae: Cyrtopodiinae). Revista Brasileira de Botânica 29: 251-258.

Mitchell, R.J.; Irwin, R.E.; Flanagan, R.J. \& Karron, J.D. 2009a. Ecology and evolution of plant-pollinator interactions. Annals of Botany 103: 1355-1363.

Mitchell, R.J.; Flanagan, R.J.; Brown, B.J.; Waser, N.M. \& Karron, J.D. 2009b. New frontiers in competition for pollination. Annals of Botany 103: 1403-1413.

Miyake, T. \& Inoue, K. 2003. Character displacement in style length between pollinator-sharing Clerodendrum trichotomum and C. izuinsulare (Verbenaceae). Plant Systematics and Evolution 243: 31-38.

Mode, C.J. 1958. A mathematical model for the coevolution of obligate parasites and their hosts. Evolution 12: 158-165.

Moody-Weis, J.M. \& Heywood, J.S. 2001. Pollination limitation to reproductive success in the Missouri evening primrose Oenothera macrocarpa (Onagraceae). American Journal of Botany 88: 1615-1622.

Moré, M.; Amorim, F.W.; Benitez-Vieyra, S.; Medina, A.M.; Sazima, M. \& Cocucci, A.A. 2012. Armament imbalances: match and mismatch in plant-pollinator traits of highly specialized long-spurred orchids. PLoS One 7: e41878.

Morgan, M.T.; Wilson, W.G. \& Knight, T.M. 2005. Plant population dynamics, pollinator foraging, and the selection of self-fertilization. The American Naturalist 166: 169-183.
Muchhala, N. \& Potts, M.D. 2007. Character displacement among bat-pollinated flowers of the genus Burmeistera: analysis of mechanism, process and pattern. Proceedings Biological sciences / The Royal Society 274: 2731-2737.

Naeem, S. \& Li, S. 1997. Biodiversity enhances ecosystem reliability. Nature 390: 507-509.

Newman, E.; Anderson, B. \& Johnson, S.D. 2012. Flower colour adaptation in a mimetic orchid. Proceedings of the Royal Society B: Biological Sciences 279: 2309-2313.

Ollerton, J. \& Cranmer, L. 2002. Latitudinal trends in plant-pollinator interactions: are tropical plants more specialised? Oikos 98: 340-350.

Ollerton, J.; Killick, A.; Lamborn, E.; Watts, S. \& Whiston. M. 2007. Multiple meanings and modes: on the many ways to a generalist flower. Taxon 56: 717-728.

Ollerton, J.; Alarcón, R.; Waser, N.M.; Price, M.V.; Watts, S.; Cranmer, L.; Hingston, A.; Peter, C.I.; \& Rotenberry, J. 2009. A global test of the pollination syndrome hypothesis. Annals of Botany 103: 14711480

Ollerton, J.; Winfree, R. \& Tarrant, S. 2011. How many flowering plants are pollinated by animals? Oikos 120: 321-326.

Oelschlägel B.; Nuss M.; von Tschirnhaus M.; Pätzold, C.; Neinhuis, C.; Dötterl, S. \& Wanke, S. 2015. The betrayed thief - the extraordinary strategy of Aristolochia rotunda to deceive its pollinators. New Phytol 206: 342-351.

Ordano, M.; Fornoni, J.; Boege, K. \& Domínguez, C.A. 2008. The adaptive value of phenotypic floral integration. New Phytologist 179: 1183-1192.

Padilla, F.M. \& Pugnaire, F.I. 2006. The role of nurse plants in the restoration of degraded environments. Frontiers in Ecology and the Environment 4: 196202.

Pannell, J.R. \& Barrett, S.C.H. 1998. Baker's law revisited: reproductive assurance in a metapopulation. Evolution 52: 657-668.

Pannell, J.R.; Auld, J.R.; Brandvain, Y.; Burd, M.; Busch, J.W.; Cheptou, P.O.; Conner, J.K.; Goldberg, E.E.; Grant, A.G.; Grossenbacher, D.L.; Hovick, S.M.; Igic, B.; Kalisz, S.; Petanidou, T.; Randle, A.M.; de Casas, R.R.; Pauw, A.; Vamosi, J.C. \& Winn, A.A. 2015. The scope of Baker's law. New Phytologist 208: 656-667.

Parker, G.A. 1970. Sperm competition and its evolutionary consequences in the insects. Biological Reviews of the Cambridge Philosophical Society 45: 525-567.

Parker, G.A. 1979. Sexual selection and sexual conflict. In: Blum, M.S. \& Blum, N.A. (eds.). Sexual selection and reproductive competition in insects. Academic Press. New York. Pp. 123-166.

Pauw, A. 2006. Floral syndromes accurately predict pollination by a specialized oil-collecting bee (Rediviva peringueyi, Melittidae) in a guild of South African 
orchids (Coryciinae). American Journal of Botany 93: 917-926

Pélabon, C.; Armbruster, W.S. \& Hansen, T.F. 2011. Experimental evidence for the Berg hypothesis: Vegetative traits are more sensitive than pollination traits to environmental variation. Functional Ecology 25: 247-257.

Peter, C.I. \& Johnson, S.D. 2008. Mimics and magnets: the importance of color and ecological facilitation in floral deception. Ecology 89: 1583-1595.

Pfennig, D.W. \& Pfennig, K.S. 2010. Character displacement and the origins of diversity. The American Naturalist 176: S26-S44.

Price, P.W. 2002. Macroevolutionary theroy on macroecological patterns. Cambridge University Press, Cambridge. 302p.

Proctor, M.; Yeo, P. \& Lack, A. 1996. The natural history of pollination. Timber Press, Portland. 487p.

Pyke, G.H. 1978. Optimal foraging in hummingbirds: testing the marginal value theorem. American Zoology 18: 739-752.

Raguso, R.A. 2008a. Start making scents: the challenge of integrating chemistry into pollination ecology. Entomologia Experimentalis et Applicata 128: 196-207.

Raguso, R.A. 2008b. Wake up and smell the roses: the ecology and evolution of floral scent. Annual Review of Ecology, Evolution, and Systematics 39: 549-569.

Real, L.A. 1981. Uncertainty and pollinator-plant interactions: the foraging behavior of bees and wasps on artificial flowers. Ecology 629: 20-26.

Rech A.R.; Kayna A.; Oliveira P.E. \& Machado I.C. 2014. Biologica da polinização. Editora Projeto Cultural, Brasília. 532p.

Rech, A.R. \& Brito, V.L.G. 2012. Mutualismos extremos de polinização: história natural e tendencias evolutivas. Oecologia australis 16: 297-310.

Rech, A.R. \& Westerkamp, C. 2014. Biologia da polinização: uma síntese histórica. In: Rech, A.R.; Agostini, K.; Oliveira, P.E. \& Machado, I.C. (eds.). Biologia da Polinização. Pp 27-43.

Richards, A.J. 1986. Plant breeding systems. George Allen \& Unwin, London. 529p.

Ricklefs, R.E. 2010. A economia da natureza. Guanabara Koogan, Rio de Janeiro. 572p.

Rodger, J.G.; van Kleunen, M. \& Johnson, S.D. 2013. Pollinators, mates and Allee effects: the importance of self-pollination for fecundity in an invasive lily. Functional Ecology 27: 1023-1033

Sandel, B.; Arge, L.; Dalsgaard, B.; Davies, R.G.; Gaston, K.J.; Sutherland, W.J. \& Svenning, J.C. 2011. The influence of late Quaternary climatechange velocity on species endemism. Science 334: 660-664.

Santamaría, L. \& Rodríguez-Gironés, M.A. 2015. Are flowers red in teeth and claw? Exploitation barriers and the antagonist nature of mutualisms. Evolutionary Ecology 29: 311-322.

Schaefer, H.M. \& Ruxton, G.D. 2009. Deception in plants: mimicry or perceptual exploitation? Trends in Ecology \& Evolution 24: 676-685.

Schärer, L., Janicke, T. \& Ramm, S.A. 2015. Sexual Conflict in Hermaphrodites. Cold Spring Harbor Perspectives in Biology, 7: a017673.

Schemske, D.W.; Mittelbach, G.G.; Cornell, H.V.; Sobel, J.M. \& Roy, K. 2009. Is there a latitudinal gradient in the importance of biotic interactions? Annual Review of Ecology, Evolution and Systematics 40: 245-269.

Schiestl, F.P. \& Johnson, S.D. 2013. Pollinator-mediated evolution of floral signals. Trends in Ecology \& Evolution 28: 307-315.

Schindler, D.E.; Hilborn, R.; Chasco, B.; Boatright, C.P.; Quinn, T.P.; Rogers, L.A. \& Webster, M.S. 2010. Population diversity and the portfolio effect in an exploited species. Nature 465: 609-612.

Schleuning, M.; Fründ, J.; Klein, A.M.; Abrahamczyk, S.; Alarcón, R.; Albrecht, M.; Andersson, G.K.; Bazarian, S.; Böhning-Gaese, K. \& Bommarco, R. 2012. Specialization of mutualistic interaction networks decreases toward tropical latitudes. Current Biology 22: 1925-1931.

Sletvold, N. \& Grindeland, J.M. 2008. Floral herbivory increases with inflorescence size and local plant density in Digitalis purpurea. Acta Oecologica 34: 21-25.

Snow, A.A. \& Lewis, P.O. 1993. Reproductive traits and male fertility on plants: empirical approaches. Annual Review of Ecology and Systematics 24: 331-35.

Stanton, M.L. 1994. Male-male competition during pollination in plant populations. American Naturalist 144: S40-68.

Stebbins, G.L. 1970. Adaptive radiation of reproductive characteristics in angiosperms, I: pollination mechanisms. Annual Review of Ecology and Systematics 1: 307-326.

Stephens, P.A.; Sutherland, W.J. \& Freckleton, R.P. 1999. What is the Allee Effect? Oikos 87: 185-190.

Stephenson, A.G. \& Bertin, R.I. 1983. Male competition, female choice, and sexual selection in plants. in Real, L. Pollination biology. Academic Press, Orlando. Pp. 109-149.

Svenning, C.J. \& Skov, F. 2007. Ice age legacies in the geographical distribution of tree species richness in Europe. Global Ecology and Biogeography 16: 234-245.

Takebayashi, N. \& Morrell, P.L. 2001. Is self-fertilization an evolutionary dead end? Revisiting an old hypothesis with genetic theories and macroevolutionary approach. American Journal of Botany 88: 11431150 .

Thibaut, L.M. \& Connolly, S.R. 2013. Understanding diversity-stability relationships: towards a unified model of portfolio effects. Ecology Letters 16: 140-150. 
Thompson, J.N. 1994. The coevolutionary process. University of Chicago Press, Chicago. 383p.

Thorp, R.W. 2000. The collection of pollen by bees. Plant Systematics and Evolution 222: 211-223.

Tripp, E.A. \& Manos, P.S. 2008. Is floral specialization an evolutionary dead-end? pollination system transitions in Ruellia (Acanthaceae). Evolution 62: 1712-1737.

Tilman, D. 1982. Resource competition and community structure. Princeton University Press, Princeton. 296p.

Vallejo-Marín, M.; Dorken, M.E. \& Barrett, S.C.H. 2010. The ecological and evolutionary consequences of clonality for plant mating. Annual Review of Ecology, Evolution and Systematics 41: 193-213.

Vallejo-Marín, M.; Manson, J.S.; Thomson, J.D. \& Barrett, S.C.H. 2009. Division of labour within flowers: heteranthery, a floral strategy to reconcile contrasting pollen fates. Journal of Evolutionary Biology 22: 828-839.

van Dijk, P.J. \& Vijverberg, K. 2005. The significance of apomixis in the evolution of the angiosperms: a reappraisal. In: Bakker, F.; Chatrou, L.; Gravendeel, B. \& Pelser, P. 2005. Plant species-level systematics: new perspectives on pattern and process. A.R.G. Gantner Verlag, Michigan. Pp. 101-116.

Vázquez, D.P. \& Stevens, R.D. 2004. The latitudinal gradient in niche breadth: concepts and evidence. American Naturalist 164: E1-E19.

Vázquez, D.P. 2005. Degree distribution in plant-animal mutualistic networks: forbidden links or random interactions? Oikos 108: 421-426.
Vázquez, D.P.; Lamáscolo, S.B.; Belén-Maldonado, M.; Chacoff, N.P.; Dorado, J.; Stevani, E.L. \& Vitale, N.L. 2012. The strength of plant-pollinator interactions. Ecology 93: 719-725.

Vizentin-Bugoni, J.; Maruyama, P.K. \& Sazima, M. 2014. Processes entangling interactions in communities: forbidden links are more important than abundance in a hummingbird-plant network. Proceedings of the Royal Society of London B 281: 1-8.

Waddington, K.D. \& Holden, L.R. 1979. Optimal foraging: on flower selection by bees. The American Naturalist 114: 179-196.

Waser, N.M. \& Ollerton, J. 2006. Plant-pollinator interactions: from specialization to generalization. University of Chicago Press, Chicago. 488p.

Waser, N.M.; Ollerton, J. \& Erhardt, A. 2011. Typology in pollination: lessons from an historical critique. Journal of Pollination Ecology 3: 1-7.

Weiner, J.; Campbell, L.G.; Pino, J. \& Echarte, L. 2009. The allometry of reproduction within plant populations. Journal of Ecology 97: 1220-1233.

Westerkamp, C. 1996. Pollen in bee-flower relations some considerations on melittophily. Botanica Acta 109: 325-332.

Willson, M.F. \& Burley, N. 1983. Mate choice in plants: tactics, mechanisms, and consequences. Princeton University Press, Princeton. 264p.

Wright, S.I.; Kalisz, S. \& Slotte, T. 2013. Evolutionary consequences of self-fertilization in plants. Proceedings of the Royal Society B: Biological Sciences 280: 20130133. 